\title{
Predictors of changes in quality of life among older people: comparison between the UK and Taiwan
}

\author{
Chih-Ping Li ${ }^{\mathrm{a}}$ \\ Department of Health Industry Management, Kainan University, Taoyuan 33857, Taiwan
}

\begin{abstract}
The purpose of this study was to compare the factors affecting change in quality of life in old age in the UK and Taiwan. Data were derived from the 1989 and 1993 waves of two nationally representative samples: the Nottingham Longitudinal Study of Activity and Ageing (NLSAA) in the UK and the Survey of Health and Living Status of the Elderlyin Taiwan (SHLSET). Quality of life was assessed using comparable measures of life satisfaction. Analysis consisted of stepwise multiple regressions, Mann-Whitney U, Chi-square, and multivariate logistic regression models to identify the factors related to changes in quality of life.There are common variables in that participants in both studies selected the factors "better perceived health relative to peers" and "improvement in self-rated health" in the stepwise regression models. Additional variables (e.g., age in the UK and satisfaction with income, walking difficulties, and having a TV or radio in Taiwan) were selected individually at different time periods in the two studies.Related to these various factors and change in quality of life, this research has helped to identify the prospect of clarifying the most important factors affecting the change in quality of life among older people in the two countries.
\end{abstract}

\section{Introduction}

Quality of life is a broad, multidimensional concept, which may be affected by such objective conditions and subjective experiences as physical functioning, social factors, perceived health, and psychological well-being. Understanding which factors affect changes in quality of life the most may be important in determining ways to improve life satisfaction as a large percentage of the population reaches their senior years.

The aging population and life expectancy are increasing, but a variety of factors may affect overall quality of life during later life [1]. In essence, older people tend to experience general health problems, difficult financial situations, limited social relationships, disruptive changes in their roles in society, loss of basic functional ability, and anxiety as they age and approach the end of life. Consequently, such problems have become major concerns in the field of gerontology and for health authorities in many countries.

Older people frequently perceive certain factors as being of greater importance to their overall quality of life. A variety of factors (morale, loneliness, physical and mental health, social engagement, etc.) may contribute to, and affect, a change in quality of life, but a key question is this: "Which

\footnotetext{
${ }^{\mathrm{a}}$ Corresponding author : luciali@mail.knu.edu.tw
} 
factors are the most important?" This study seeks to provide a better understanding of what factors affect change in quality of life among older people.

To understand the differences in predictors of change in life satisfaction, the author proceeded to (1) identify the factors that predict a change in life satisfaction among older people in the UK and Taiwan and (2) understand how these factors are different between the two countries.

\section{Methods}

\subsection{Participants and ethics}

Data were derived from the Nottingham Longitudinal Study of Activity and Ageing (NLSAA) and the Survey of Health and Living Status of the Elderly in Taiwan (SHLSET). Ethics was approved by the Department of Information Studies, in accordance with the University of Sheffield research ethics policies.

The NLSAA study was set up in 1985, with follow-up interviews in 1989 and 1993 [2, 3]. Of 8,409 older people (aged 65 and over) in three areas of Nottingham, UK, 1,299 were randomly selected for the interview, and 1,042 agreed to participate in the 1985 baseline survey (response rate $=$ $80.2 \%$ ), of whom 690 were still alive and participated in the follow-up survey in 1989 (response rate $=$ $88.3 \%)[3,4]$.

The SHLSET was jointly conducted by the Taiwan Provincial Institute of Family Planning, the Population Studies Center (PSC), and Institute of Gerontology at the University of Michigan [5]. The baseline survey of SHLSET was conducted in 1989. The sample size was 4,412 people (age 60 years and over), and the response rate was $91.8 \%$, with 4,049 participants interviewed.

To obtain comparable samples from the two studies, individuals born before 30 April 1920 and who were interviewed in 1989 were included in this study. The final samples contained 690 individuals from the NLSAA and 1,438 individuals from SHLSET.

\subsection{Survey assessments}

The data collection instrument for the NLSAA was a structured interview questionnaire, composed of 161 questions that addressed demographics, mobility, physical health, psychological health (cognitive status, depression, and morale), social functioning, use of health and personal social services, customary physical activity, and physical measurements [2].

The SHLSET questionnaire was composed of eight sections such as marital history and other background characteristics; household schedule, social and economic exchanges; health, health care utilization, and behaviors; occupational history; activities and general attitudes; residence history; economics/financial well-being, andemotional and instrumental support[3, 4].

The NLSAA and the SHLSET both used shortened versions of the Life Satisfaction Index, a commonly used measure of well-being and morale in studies of older people [6]. In the NLSAA, the 13-item Life Satisfaction Index-Z (LSIZ) [7] was used. For the SHLSET, the life satisfaction scale was assessed using a 10-item scale in 1989 and a 4-item scale in 1993 [8]. For comparative purposes, a common scoring system for each response category and question across the two studies was adopted and the scores for both scales were standardized to a 100-point scale.

Other variables from the two studies were compared, and variables with similar questions and response categories were harmonized using established methods $[9,10]$ to create new variables that cover demographics and socioeconomics, physical and mental health, and social activity [11].

\subsection{Data analyses}

Change in standardized life satisfaction score was the dependent variable used in the third part of the analyses. The change in life satisfaction was produced from the life satisfaction standardized score in 
1989 minus the life satisfaction standardized score in 1993, so that a positive value indicated an increase in life satisfaction from 1989 to 1993 and a negative value indicated a decrease in life satisfaction from 1989 to 1993.

Twenty-five independent variables from the data harmonization process were used: gender, marital status, living status, number of people living in the household, socioeconomic class, satisfaction with income, self-rated health, perceived health relative to peers, smoking, arthritis or rheumatism, heart trouble, stomach problems, dizziness, high blood pressure, urinary incontinence, walking difficulties, use of a walking aid, loneliness, standardized SAD score, friends, reading newspapers or journals, attending a religious group, attending some other club or organization, having a TV or radio, and having a pet. These independent variables were assessed to determine whether they were associated with a change in life satisfaction index between 1989 and 1993. For example, a new response category was created as "no change," "became widowed or separated/divorced," "became married," and "two changes" for the marital status variable according to the difference factors between 1989 and 1993. With regard to diseases, for instance, a new response category of "new problem," "no change," and "no longer a problem in 1993" was generated for the arthritis or rheumatism problem variable. However, the age variable used was the participants' age in 1993, the gender and socioeconomic class variables were those used in 1989, and the standardized SAD score used the difference between 1989 and 1993 (the score in 1989 minus the score in 1993).

Change in life satisfaction was analyzed in two ways: first as a continuous variable, for which stepwise multiple regression with criteria as probability-of-F-to-enter $<=0.050$ and probability-of-Fto-remove $>=0.100$ was used to identify the factors related to life satisfaction as a continuous variable in the two studies separately.

To identify which factors affect change in life satisfaction, the variable "change in standardized LSI score" was dichotomized into "decrease" and "no change/increase" in life satisfaction and, and using both bivariate and multivariate analysis, assessed for the relationship between this new dichotomized dependent variable and each of the harmonized variables from the 1989 and 1993 surveys.

Bivariate analysis was conducted using the Mann-Whitney $\mathrm{U}$ test for continuous independent variables. Pearson chi-square analysis was used for categorical independent variables. In each instance, it was assessed whether a relationship between the variables was significant or not. For multivariate analysis, logistic regression analysis was performed to identify the most important factors affecting change in life satisfaction.

Because of the large number of tests carried out, $\alpha$ was set to 0.01 in order to reduce the likelihood of a Type I error. However, any variables that had a $p$ value of 0.05 or less were included in the logistic regression model to ensure no potentially important factors were omitted. Even though these variables were significantly associated with low/high life satisfaction, they may have an important modifier effect on the other variables.

\section{Results}

The one-sample Kolmogorov-Smirnov test was used to test whether the distribution for the change in the standardized LSI score was normal. The finding shows that change in the standardized LSI score for the NLSAA study, the SHLSET study, and the combined dataset exhibited a distribution that was not normal. In the SHLSET study, one person had a change of 100 points because he/she had a score of 0 points in 1989. On the other hand, there were two people who had a change of -100 points became they went from 100 points in 1989 to 0 points in 1993. This may well have happened because these two people could not answer the four questions in the LSI in 1993. On comparing the mean score for the change in the standardized LSI, it was found that older people in the NLSAA study had a smaller reduction in the standardized LSI score than the older people in the SHLSET study.

The change in the standardized life satisfaction score in the NLSAA study was more peaked than would be expected for a normal distribution. 


\subsection{Change in life satisfaction as a continuous variable}

Table 1 shows the results of stepwise regression analysis to identify predictors of change in life satisfaction in the NLSAA study between 1989 and 1993. Stepwise regression was conducted and the final combination of variables was perceived health relative to peers, self-rated health, and age in 1993. This combination of variables significantly predicted change in life satisfaction, $F_{(3,306)}=13.647$, $p<0.001$, with all three variables significantly contributing to the model.

Three variables were included in the final model to predict change in standardized life satisfaction score in the NLSAA study, and this model explained $10.9 \%$ of the variance in change in life satisfaction. The change in perceived health relative to peers variable (6.7\% of the variance) explained the largest share of the variance, followed by change in self-rated health $(3.0 \%)$ and age $(1.2 \%)$. In the regression model 3, the relationship between the age (1993) variable and the change in standardized life satisfaction score was negative (-0.122), indicating that older people had a reduction in life satisfaction.

Table 1. Factors affecting change in life satisfaction in the NLSAA $(\mathrm{N}=381)$.

\begin{tabular}{llcccccc}
\hline Model & $\mathrm{r}$ & $\begin{array}{c}\text { Adjusted } \\
\mathrm{R}^{2}\end{array}$ & $\begin{array}{c}\text { Change } \\
\text { in } \mathrm{R}^{2}\end{array}$ & Beta & 95\% CI & $p$ value \\
\hline 1 & $\begin{array}{l}\text { Change in perceived health } \\
\text { relative to peers }\end{array}$ & 0.265 & 0.067 & - & 0.265 & $4.818-11.438$ & $<0.001$ \\
2 & Change in self-rated health & 0.321 & 0.097 & 0.030 & 0.188 & $2.028-7.844$ & 0.001 \\
3 & Age in 1993 & 0.344 & 0.109 & 0.012 & -0.122 & $-0.958--0.070$ & 0.023
\end{tabular}

Note: The excluded variables include gender, marital status, socioeconomic class, number of people living in household, satisfaction with income, smoking, arthritis or rheumatism, heart trouble, stomach problems, giddiness problem, high blood pressure, urinary incontinence, walking problem, walking aid, loneliness, standardized SAD score, having a pet, having friends, reading newspapers or journals/magazines, attending a religious group, and participating in a club or organization.

Table 2. Factors affecting change in life satisfaction in SHLSET $(\mathrm{N}=488)$.

\begin{tabular}{llcccccc}
\hline Model & $\mathrm{r}$ & $\begin{array}{c}\text { Adjusted } \\
\mathrm{R}^{2}\end{array}$ & $\begin{array}{c}\text { Change in } \\
\mathrm{R}^{2}\end{array}$ & Beta & $95 \% \mathrm{CI}$ & $p$ value \\
\hline 1 & Change in self-rated health & 0.258 & 0.064 & - & 0.258 & $7.517-15.458$ & $<0.001$ \\
2 & 0.308 & 0.091 & 0.027 & 0.169 & $4.499-14.372$ & $<0.001$ \\
& $\begin{array}{l}\text { Change in satisfaction with } \\
\text { income }\end{array}$ & 0.334 & 0.106 & 0.015 & -0.135 & $-15.816--3.116$ & 0.004 \\
$\begin{array}{l}\text { Change in walking } \\
\text { problems }\end{array}$ & 0.352 & 0.116 & 0.010 & 0.128 & $1.387-10.736$ & 0.011 \\
4 & $\begin{array}{l}\text { Change in perceived health } \\
\text { relative to peers }\end{array}$ & 0.368 & 0.126 & 0.010 & 0.107 & $2.149-20.501$ & 0.016 \\
\hline
\end{tabular}

Note: The excluded variables include age, gender, marital status, socioeconomic class, number of people living in household, smoking, arthritis or rheumatism, heart trouble, stomach problems, giddiness problem, high blood pressure, urinary incontinence, walking aid, loneliness, having a pet, having friends, reading newspapers or journals/magazines, attending a religious service, and participating in a club or organization.

Table 2 presents the results of stepwise regression analysis to identify predictors of change in life satisfaction in the SHLSET study between 1989 and 1993. The variables are included in the final model. The adjusted $\mathrm{R}$ squared value was 0.126 , indicating that $12.6 \%$ of the variance in change in life satisfaction was explained by the five variables in the model. Self-rated health $(6.4 \%$ of the variance) explained more than half of this variance, followed by satisfaction with income (2.7\%), walking problems $(1.5 \%)$, perceived health relative to peers $(1.0 \%)$, and having a TV or radio (1.0\%). In the regression model 3 , the relationship between the walking difficulty variable and the change in standardized life satisfaction score was negative $(-0.135)$, implying that a person with walking 
problems had a reduced life satisfaction score. This combination of variables significantly predicted change in life satisfaction, $\mathrm{F}_{(5,450)}=14.100, p<0.001$, with all five variables contributing significantly to the model.

In the SHLSET study, five variables contributed significantly to changes in life satisfaction: higher self-rated health, satisfaction with income, perceived health better than peers, and having a TV or radio were related to an increase in life satisfaction, whereas walking difficulties predicted a negative change in life satisfaction.

\subsection{Change in life satisfaction as a categorical variable}

In the NLSAA survey, the results of Mann-Whitney $U$ tests assessed the relationship between the continuous independent variables and change in life satisfaction between 1989 and 1993. MannWhitney $U$ tests revealed a statistically significant difference in the mean rank for the change in standardized SAD score $(\mathrm{Z}$ statistic $=-4.663 ; p<0.001)$ between those with no change or an increase in life satisfaction versus those with a decrease. Older people who experienced a decrease in life satisfaction were more depressed than the others.

The results of the Mann-Whitney $U$ tests also assessed the relationship between the continuous independent variables and change in life satisfaction between 1989 and 1993 for the SHLSET survey.The results were similar to those of the NLSAA study. Older people who had higher levels of depression $(\mathrm{Z}=-3.660 ; p<0.001)$ were more likely to experience a decrease in life satisfaction.

The association was between change in independent variables (except gender and socioeconomic class) and change in life satisfaction between 1989 and 1993 in the NLSAA study. A significant association existed between changes in life satisfaction and changes in self-rated health $\left(\chi^{2}=4.199\right.$; $p=0.040)$, perceived health relative to peers $(\chi=7.607 ; p=0.006)$, and arthritis or rheumatism $\left(\chi^{2}=4.033\right.$; $p=0.045)$. Older people who rated their health as worsening $(60.7 \%)$, perceived their health relative to peers as becoming worse (66\%), and no change in arthritis or rheumatism (54\%) were associated with decreased life satisfaction.

The association was between changes in independent variables and change in life satisfaction between 1989 and 1993 in the SHLSET dataset. There was a significant association between changes in life satisfaction and change in self-rated health $\left(\chi^{2}=9.855 ; p=0.002\right)$, perceived health relative to peers $\left(\chi^{2}=11.746 ; p=0.001\right)$, having a TV or radio $\left(\chi^{2}=4.136 ; p=0.042\right)$, reading a newspaper or journal $\left(\chi^{2}=6.013 ; p=0.014\right)$, and participating in a club or organization $\left(\chi^{2}=4.064 ; p=0.044\right)$.Participants in Taiwan whose level of self-rated health had decreased $(63.6 \%)$, who reported that their health relative to their peers became worse $(65.5 \%)$, who no longer had a TV or radio $(62.5 \%)$, who no longer read a newspaper or journal/magazine $(70.7 \%)$, and who no longer attended a club or organization $(76.7 \%)$ were more likely to have a decrease in life satisfaction.

Table 3. Selected variables associated with reduced life satisfaction in the NLSAA study (1989-93).

\begin{tabular}{llccc}
\hline $\begin{array}{l}\text { Variable } \\
\text { (reference category) }\end{array}$ & Category & Odds ratio & $95 \%$ CI & $p$ value \\
\hline Self-rated health & & & & 0.052 \\
(No change) & An improvement & 0.740 & $0.427-1.284$ & 0.284 \\
& A worsening & 1.465 & $0.923-2.324$ & 0.105 \\
Perceived health relative to peers & & & & 0.074 \\
(No change) & Better health & 1.058 & $0.615-1.820$ & 0.838 \\
& Worse health & 1.797 & $1.080-2.990$ & 0.024 \\
& & & & 0.113 \\
Arthritis or rheumatism(No change) & No longer present & 0.682 & $0.365-1.276$ & 0.231 \\
& New problem & 0.555 & $0.299-1.030$ & 0.062 \\
& & 1.031 & $1.013-1.050$ & 0.001 \\
\hline
\end{tabular}


Logistic regression models were used to identify which selected variables were the best predictors of change in life satisfaction from 1989 to 1993 using data from each of the two studies separately.

The selected independent variables were associated with change in life satisfaction between 1989 and 1993 in the NLSAA study. When all the selected variables were considered together, they were significantly associated with changes in life satisfaction $\left(\chi^{2}=33.497 ; d f=7 ; p<0.001\right)$. Table 3 shows a significant association between negative or lower changes in life satisfaction and level of depression $(p=0.001)$.People who had higher levels of depression $(\mathrm{OR}=1.031)$ were more likely to experience decreased life satisfaction. The other variables did not have a statistically significant association.

Table 4 presents the selected variables associated with change in life satisfaction between 1989 and 1993 in the SHLSET dataset.The overall model with all the variables entered was significant $\left(\chi^{2}=55.169 ; d f=11 ; p<0.001\right)$. As shown in Table 4 , there was a significant association between changes in life satisfaction and self-rated health $(p=0.016)$ and perceived health relative to peers $(p=0.009)$; the same association was true with change in levels of depression $(p=0.018)$.Compared to people who experienced no change, people whose self-rated health diminished $(\mathrm{OR}=1.437)$ were more likely to have decreased life satisfaction; and people whose health relative to their peers worsened $(\mathrm{OR}=1.631)$ were more likely to experience decreased life satisfaction. People who had higher levels of depression $(\mathrm{OR}=1.009)$ were more likely to experience decreased life satisfaction.

Table 4. Selected variables associated with reduced life satisfaction in SHLSET (1989-93).

\begin{tabular}{llccc}
\hline Variable (reference category) & Category & Odds ratio & $95 \%$ CI & $p$ value \\
\hline Self-rated health & & & & 0.016 \\
(No change) & An improvement & 0.826 & $0.579-1.177$ & 0.289 \\
& A worsening & 1.437 & $1.012-2.041$ & 0.043 \\
Perceived health relative to peers & & & & 0.009 \\
(No change) & Better health & 0.872 & $0.607-1.252$ & 0.457 \\
& Worse health & 1.631 & $1.140-2.333$ & 0.007 \\
Change in standardized SAD score & & 1.009 & $1.002-1.017$ & 0.018 \\
Newspaper or journal & & & & 0.133 \\
(No change) & No longer read & 0.833 & $0.425-1.633$ & 0.596 \\
& Started reading & 1.874 & $0.983-3.571$ & 0.056 \\
TV or radio & & & & 0.069 \\
(No change) & No longer have & 1.900 & $0.799-4.516$ & 0.146 \\
& Acquired & 1.730 & $0.971-3.083$ & 0.063 \\
Club or organization & & & & 0.193 \\
(No change) & No longer attending & 0.887 & $0.396-1.987$ & 0.771 \\
& Became able to attend & 2.250 & $0.922-5.490$ & 0.075 \\
\hline
\end{tabular}

\section{Discussion}

The purpose of this study is to understand which factors are associated with a change in the quality of life among older people, and understanding the changes in the quality of life and the factors that predict these changes may help to develop ways to improve the quality of life of older people. There are common variables in that in the stepwise regression models, participants selected the factors "better perceived health relative to peers" and "improvement in self-rated health" in both studies. Additional variables (e.g., age in the UK and satisfaction with income, walking difficulties, and having a TV or radio in Taiwan) were selected individually at different time periods in the two studies. These variables tended to be selected in the regression model, and account for a small proportion of the variance, and therefore, are related to changes in life satisfaction. The study location variable was identified as a predictor of change in life satisfaction; however, this result suggests that older people in 
the UK tended to have decreased life satisfaction as they aged when compared with their counterparts in Taiwan.

In the logistic regression models, an increased standardized SAD score was related to decreased life satisfaction in the two studies, and worsened self-rated health and worsened perceived health relative to peers were associated with decreasing life satisfaction among older people in the SHLSET. These results suggested that mental health was related to a change in life satisfaction among older people in both countries; however, in the case of people in Taiwan, change in perceived health relative to their peers had an effect on life satisfaction.

This research examined change in life satisfaction among older people from 1989 to 1993 and compared the two studies from the UK and Taiwan.Perceived better health relative to peers and improvement in self-rated health were associated with increased life satisfaction in both stepwise regression models. Additional variables were selected individually at different points in the two studies. These variables were selected in the regression models and account for a small proportion of the variances, and therefore, have a relationship with change in life satisfaction. In the logistic regression models, an increased standardized SAD score was related to decreased levels of life satisfaction in the two studies, and worsened self-rated health and worsened perceived health relative to peers were associated with decreased life satisfaction among older people in SHLSET.

The result agreed with previous studies in which it was determined that age, self-rated health, and social activities are associated with changes in life satisfaction $[12,13]$, but very little is known about the relationship between change in depression level and change in life satisfaction. People who grew older that rated health worse, perceived health worse relative to peers, and had new walking difficulties were more likely to show a decreased life satisfaction level. As discussed earlier, self-rated health and perceived health relative to peers were difficult to assess and might confound other unmeasured variables. Although these variables had only minor variances identified as affecting a change in life satisfaction, small life satisfaction changes may be statistically significant, especially with large studies.

A limitation of this research is that it did not explore how many changes in the standardized LSI scores could be interpreted in terms of small, moderate, or large changes in life satisfaction as reported by older people. Further research is needed to understand what factors affect the different levels of life satisfaction.

Another limitation, as with most research of this kind, is that it is likely that certain participant characteristics, related to both dependent and independent variables (so-called "confounders"), were not examined in the research. It must be kept in mind that the presence of such confounding variables - known or unknown - could explain at least part of the relationships detected in this research. For example, it was not possible to assess certain disease states. It is possible that some disease states that were not examined (e.g., alcohol abuse) could be related to quality of life and a variety of other factors; and not examining these conditions may limit the extent to which these findings can be interpreted.

Future studies could resolve some of the more minor shortcomings of the combined data sets used in the present analyses. In the future, measures incorporating the length, breadth, and profundity of quality of life in different countries would significantly improve the understanding of ageing populations.

\section{Conclusions}

Lifesatisfaction is a dynamic process that changes according to an individual's circumstances [14]. Our results support this contention among older people, who appeared to evaluate their overall life satisfaction based upon such circumstances as their financial status or whether they had a TV or radio. Financial strain and low income have been found to be key factors causing low life satisfaction among older people $[15,16]$. However, a decreased income might have had a stronger effect in decreasing the levels of life satisfaction in old age. 
The present research indicates what factors are associated with life satisfaction among older people. There are numerous determinants of life satisfaction in older people, and most of these hold true in both the United Kingdom and in Taiwan. Aspects of physical health are important, as are one's mobility and one's mental health. Satisfaction with one's personal financial status also appears to play a significant role. In addition, these relationships largely seem to hold true over time. However, the numerous interactions between these various contributors to health are complicated. In other words, what is the horse, what is the cart, and what is the chain linking the two is not entirely clear; further research is warranted. This study has helped tease out general trends and the most important variables.

Aspects of physical and mental health were associated with changes in life satisfaction among people in the two countries. In particular, depression affected change in life satisfaction among people as they aged. Although there are few previous studies regarding change in life satisfaction, there was some evidence in this research that changes in perceived health and mobility might be associated with increased depression because depression might be a result of disease and lessen activities of daily living, thus leading to low life satisfaction.

Related to these various factors and change in quality of life is the prospect of clarifying the most important factors affecting the change in quality of life in old age. Older people in different countries may have specific problems because of local differences in conditions and life experience. A contribution of this research is improving the understanding of the factors that adversely affect people's quality of life in old age. However, such findings underscore the importance of recognizing the need for older people to maintain their quality of life. Such factors as participating in activities with others, maintaining health, developing social relationships from community-based schemes, requiring resources from social support systems are some ways that older people can maintain their quality of life and mitigate some of the factors that indicated a lower or worsening life-satisfaction as they aged.

The next major step with regards to these results is to determine why certain associations happen in these people in the UK and/or Taiwan. For instance, loneliness appears to be more common among older people in the UK. Studies will be required to test alternative explanations systematically for the influence of psychosocial variables on physiological processes and quality of life. Qualitative fieldwork to investigate how older people deal with loneliness could prove useful. In this research, although depression was a major influence on quality of life, the levels of depression did not affect the relationship between quality of life and mortality in the two studies. Even though some investigators have explored the relationship between depression and mortality, few studies have looked at the effect of depression on quality of life and its relationship to mortality: the association between these factors could be investigated in future studies.

\section{Acknowledgements}

I am grateful to Professor Kevin Morgan for access to the Nottingham Longitudinal Study of Activity and Ageing in the UK. The NLSAA was initiated with a foundation grant from the Grand Charity. Additional support for the study was provided by Help the Aged, PPP Charitable Trust and Trent Regional Health authority. This research also utilized the data set from the Survey of Health and Living Status of the Elderly in Taiwan, provided by the Bureau of Health Promotion, Department of Health Taiwan.

\section{References}

1. Y. Hellström, G. Persson, and I.R. Hallberg, Quality of life and symptoms among older people living at home, Journal of Advanced Nursing, 48, 584-593 (2004)

2. K. Morgan, The Nottingham Longitudinal Study of Activity and Ageing (NLSAA) Survey Interview and Data Catalogue (University of Sheffield, Britain, 1993)

3. K. Morgan, The nottingham longitudinal study of activity and ageing: a methodological overview, Age and Ageing, 27, 5-11 (1998) 
4. K. Morgan and P.A. Bath, Customary physical activity and psychological wellbeing: a longitudinal study, Age and Ageing, 27, 35-40 (1998)

5. Taiwan Provincial Institute of Family Planning, "1989 Survey of health and living status of the elderly in Taiwan: Questionnaire and survey design," Comparative Study of the Elderly in Four Asian Countries (Taiwan Provincial Institute of Family Planning, Population Studies Centre and Institute of Gerontology, University of Michigan, Taichung, 1989)

6. K.A. Wallace and A.J. Wheeler, Reliability generalization of the Life Satisfaction Index, Educational and Psychological Measurement, 62, 674-684 (2002)

7. K. Morgan, H.M. Dallosso, and T.H.D. Arie, Mental health and psychological well-being among the old and very old living at home, British Journal of Psychiatry, 150, 801-807 (1987)

8. C. Chen, Aging and life satisfaction, Social Indicators Research, 54, 57-79 (2001)

9. P.A. Bath, D. Deeg, and J.L. Poppelaars, The harmonisation of longitudinal data: a case study using data from cohort studies in The Netherlands and the United Kingdom, Ageing and Society, 30, 1419-1437 (2010)

10. N. Minicuci, et al., Cross-national determinants of quality of life from six longitudinal studies on aging: The CLESA project, Aging Clinical and Experimental Research, 15, 187-202 (2003)

11. C.P. Li, Thesis, University of Sheffield, Sheffield, UK (2009)

12. A. Bowling, M. Farquhar, and E. Grundy, Associations with changes in life satisfaction among three samples of elderly people living at home, International Journal of Geriatric Psychiatry, 11, 1077-1087 (1996)

13. D.K. Mroczek and A. Spiro, III, Change in life satisfaction during adulthood: findings from the Veterans Affairs Normative Aging Study, Journal of Personality and Social Psychology, 88, 189202 (2005)

14. R. Fernández-Ballesteros, Quality of life: the differential conditions, Psychology in Spain, 2, 5765 (1998)

15. E. Aurelia and B. Baldazzi, Unequal perceived quality of life among elderly Italians: different satisfaction levels in selected spheres life, Social Indicators Research, 60, 309-334 (2002)

16. K.L. Chou and I. Chi, Financial strain and life satisfaction in Hong Kong elderly Chinese: moderating effect of life management strategies including selection, optimization, and compensation, Aging and Mental Health, 6, 172-177 (2002) 